\title{
Guidance and Control Method for Vehicle with Strap-down Seeker
}

\author{
Li Fugui ${ }^{1}$, Jia Shengwei ${ }^{1}$, 2,a , Tong Zeyou ${ }^{1}$, Zhao Changjian ${ }^{1}$, Li Yahui ${ }^{1}$, Gao Feng ${ }^{1}$ and Gong Min ${ }^{1}$ \\ ${ }^{1}$ China Academy of Launch Vehicle Technology, 100076 Beijing, China \\ ${ }^{2}$ Nanjing University of Aeronautics and Astronautics, 210016 Nanjing, China
}

\begin{abstract}
Guidance and control method was presented in this paper for flight vehicle with a strap-down seeker. Firstly, an extended Kalman filter was constructed in LOS (line of sight) coordinate system to estimate the guidance signal based on the information measured by strap-down seeker and inertial measurement unit. Secondly, an optimal guidance law which could reduce the target maneuvering and guidance system dynamics was proposed. Thirdly, three-loop acceleration autopilot with pseudo-angle of attack feedback was presented to control the flight vehicle. Finally, the proposed methods were simulated under typical condition. The simulation results demonstrated that the proposed methods were valid, and good performance could be achieved by the guidance and control method with the guidance signal estimated by the extended Kalman filter.
\end{abstract}

\section{Introduction}

The strap-down seeker removed the complex gimbals and electrometrical structures compared with the traditional gimbaled seeker. And the detector was rigidly mounted on the body of vehicle. The size of the seeker could be much smaller and the response time could be much faster than gimbaled seeker. Because of these advantages, the strap-down seeker was a good new choice in the engineering [1][2]. But the strap-down seeker needed to isolate the disturbance of the body with mathematics method[3]. And the guidance signal could not been measured by the seeker directly. The Kalman filter was usually been used to estimate the guidance signal[4]. The appropriate filter method was very important.

The guidance law was the key part of the guidance system, which had important effect to the guidance performance [5-7]. The optimal guidance law could reduce the miss distance due to target maneuvering and the guidance system dynamics[7-10]. In order to use optimal guidance law, the guidance information should be estimated above all.

For the vehicle with estimated guidance signal, the acceleration command would be noisy. That demanded the autopilot should be robust as well as reliability. Many different forms of autopilot had been studied in some literatures[1,6,7,11]. To make sure the control process was smooth and reliable, the autopilot with good performance should been chosen.

In this paper, the extended Kalman filter which was constructed in polar coordinate system to estimate guidance signals with the information measured by phased array radar seeker was presented at first. Then, the optimal guidance law was proposed in practical form, and the guidance signal was from the estimated information

\footnotetext{
${ }^{\mathrm{a}}$ Corresponding author: 1fg200410792@sina.com
}

of the extended Kalman filter. Also, Three-loop autopilot with pseudo-angle of attack feedback was presented to make sure the control process was accurate and stable. Under the typical condition, the simulation was done to verify the performance of methods. In order to explain the method easily, the analysis was done in pitch plane.

\section{Guidance signal estimation in LOS coordinate system}

\subsection{State equation of extended Kalman filter}

Angle relation of strap-down seeker was represented in Fig.1. $q_{\mathrm{p}}$ was angle of LOS. $\vartheta$ was angle of pitch. $\varepsilon$ was the angle between the body axis of vehicle and LOS, and $\mathrm{R}$ was the relative distance between the flight vehicle and the target. $\vartheta$ could be measured by the inertial measurement unit. Both $\varepsilon$ and $R$ could be measured by strap-down phased array radar seeker. It was easily to get Eq.1.

$$
q_{p}=\vartheta+\varepsilon
$$

The inertial coordinate system xoy and LOS coordinate system $x_{l} o y_{l}$ could be established as Fig.2.

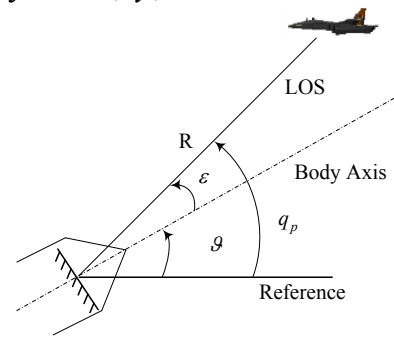

Figure 1. Angle relation of the strap-down seeker 


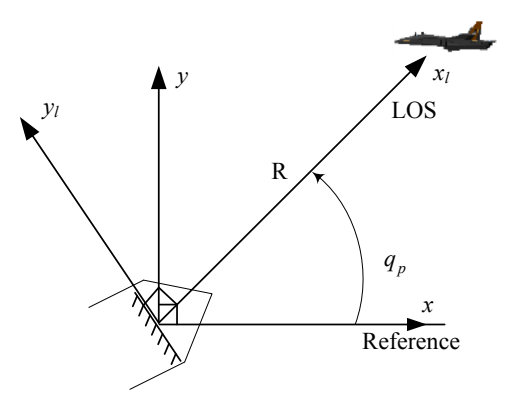

Figure 2. The inertial and LOS coordinate system

The projections of relative position between the vehicle and the target in inertial coordinate system were $x$ and $y$. Relative velocity between the vehicle and the target in inertial coordinate system were $v_{x}$ and $v_{\mathrm{y}}$. And projections of relative acceleration were $a_{x}$ and $a_{y}$. From Fig.2, it was easily to get Eq.2.

$$
\left\{\begin{array}{l}
q_{p}=\arctan (y / x) \\
R=\sqrt{x^{2}+y^{2}} \\
x=R \cos \left(q_{p}\right) \\
y=R \sin \left(q_{p}\right)
\end{array}\right.
$$

The state vector could be chosen as $\mathbf{X}=\left[q_{p}, \dot{q}_{p}, R, \dot{R}, a_{t x}^{l}, a_{t y}^{l}\right] . \dot{q}_{p}$ was angular rate of LOS. $\dot{R}$ was relative velocity along LOS. $a_{t x}^{l}$ and $a_{t y}^{l}$ were the projections of target acceleration in LOS coordinate system.

With some algebraic operation, the state equation of extended Kalman filter could be obtained as Eq.3. Where $w$ was process Gaussian white noise, and its power spectrum density was $S_{w} . a_{m x}^{l}$ and $a_{m y}^{l}$ were the projections of acceleration of missile in LOS coordinate system.

$$
\begin{aligned}
& \dot{\mathbf{X}}=\mathbf{A X}+\mathbf{G} w \\
& \mathbf{A}=\left[\begin{array}{cccccc}
0 & 1 & 0 & 0 & 0 & 0 \\
0 & -2\left(\frac{\dot{R}}{R}\right) & -\frac{a_{t y}^{l}-a_{m y}^{l}-2 \dot{R} \dot{q}_{p}}{R^{2}} & -2 \frac{\dot{q}_{p}}{R} & 0 & \frac{1}{R} \\
0 & 0 & 0 & 1 & 0 & 0 \\
0 & 2 R \dot{q}_{p} & \dot{q}_{p}^{2} & 0 & 1 & 0 \\
0 & a_{t y}^{l} & 0 & 0 & 0 & \dot{q}_{p} \\
0 & -a_{t x}^{l} & 0 & 0 & -\dot{q}_{p} & 0
\end{array}\right] \\
& \mathbf{G}=\left[\begin{array}{c}
0 \\
0 \\
0 \\
0 \\
\cos q_{p}+\sin q_{p} \\
-\sin q_{p}+\cos q_{p}
\end{array}\right]
\end{aligned}
$$

\subsection{Measurement equation}

As it was proposed before, that $\mathrm{R}$ and $\vartheta$ and $\varepsilon$ could be measured directly. With Eq.1, the measurement equation could be described as Eq.4.

$$
\begin{gathered}
\mathbf{Z}=\mathbf{C X}+v \\
\mathbf{Z}=\left[\begin{array}{c}
q_{p}{ }^{*} \\
R^{*}
\end{array}\right] \quad \mathbf{C}=\left[\begin{array}{llllll}
1 & 0 & 0 & 0 & 0 & 0 \\
0 & 0 & 1 & 0 & 0 & 0
\end{array}\right] \quad \mathbf{v}=\left[\begin{array}{c}
v_{q} \\
v_{R}
\end{array}\right]
\end{gathered}
$$

Where $v_{q}$ and $v_{R}$ were measurement Gaussian white noises. The superscript * stood for measurement value.

\subsection{Discrete extended Kalman filter}

Let $T_{\mathrm{s}}$ be the discrete time step. Based on Eq.3 and Eq.4, the discrete extended Kalman filter could be gotten as Eq.5.

$$
\left\{\begin{array}{l}
\hat{\mathbf{X}}_{k}=\boldsymbol{\Phi}_{k} \hat{\mathbf{X}}_{k-1}+\mathbf{K}_{k}\left(\mathbf{Z}_{k}-\mathbf{C} \boldsymbol{\Phi}_{k} \hat{\mathbf{X}}_{k-1}\right) \\
\mathbf{M}_{k}=\boldsymbol{\Phi}_{k} \mathbf{P}_{k-1} \boldsymbol{\Phi}_{k}^{T}+\mathbf{Q}_{k} \\
\mathbf{K}_{k}=\mathbf{M}_{k} \mathbf{C}^{\boldsymbol{T}}\left[\mathbf{C} \mathbf{M}_{k} \mathbf{C}^{\boldsymbol{T}}+\mathbf{R}_{k}\right]^{-1} \\
\mathbf{P}_{k}=\left(\mathbf{I}-\mathbf{K}_{k} \mathbf{C}\right) \mathbf{M}_{k}
\end{array}\right.
$$

The subscript $k$ stood for the current step, and $k$-1 stood for the before step. Where $\mathbf{K}_{k}$ was the gain matrix of the extended Kalman filter, $\mathbf{R}_{k}$ was the covariance matrix of the measurement noises, $\mathbf{M}_{k}$ was the covariance matrix before the update, $\mathbf{P}_{k}$ was the covariance matrix, $\mathbf{Q}_{k}$ was the discrete system noise matrix, $\mathbf{Z}_{k}$ was the discrete measurement matrix. $\boldsymbol{\Phi}_{k}$ was the discrete state transition matrix.

The state transition matrix could be derived from Eq.6.

$$
\mathbf{\Phi}(t)=e^{\mathbf{A} t}=\mathbf{I}+\mathbf{A} t+\frac{(\mathbf{A} t)^{2}}{2 !}+\ldots+\frac{(\mathbf{A} t)^{n}}{n !}+\ldots
$$

If took the first two items to approximate, then $\boldsymbol{\Phi}_{k}$ could be gotten as Eq.7.

$$
\mathbf{\Phi}_{k} \approx \mathbf{I}+\mathbf{A} T_{s}
$$

$\mathbf{Q}_{k}$ could be derived from Eq.8.

$$
\mathbf{Q}_{k}(\hat{\mathbf{X}})=\int_{0}^{T_{s}} \boldsymbol{\Phi}(t) \mathbf{G} \mathbf{S}_{w} \mathbf{G}^{\boldsymbol{T}} \boldsymbol{\Phi}^{\boldsymbol{T}}(t) d t
$$

$\mathbf{R}_{k}$ could be derived from Eq.9, where $\sigma_{q}{ }^{2}$ and $\sigma_{R}{ }^{2}$ were the variance of measurement noise $v_{q}$ and $v_{R}$.

$$
\mathbf{R}_{k}=\mathrm{E}\left(v v^{\boldsymbol{T}}\right)=\left[\begin{array}{ll}
\sigma_{q}{ }^{2} & \\
& \sigma_{R}{ }^{2}
\end{array}\right]
$$

The convergence of extended Kalman filter was sensitive to the initialization. In practical application, the estimate state of the Kalman filter was typically set to be zero. The measurement state could be set as before measured information. Then the initial state could be set as Eq. 10 .

$$
\hat{\mathbf{X}}(0)=\left[\hat{q}_{p 0}, 0, \hat{R}_{0}, \hat{\dot{R}}_{0}, 0,0\right]^{\mathrm{T}}
$$

where $\hat{q}_{p 0}, \hat{R}_{0}$ and $\hat{\dot{R}}_{0}$ were the initial estimation of the LOS angle, relative distance and relative velocity, respectively. The superscript $\wedge$ stood for estimate value. If the maximum measured error of $q_{\mathrm{p}}$ and $R$ were $\Delta q$ and $\Delta R$. The maximum estimated error of $\dot{q}_{p}$ and $\dot{R}$ were $\Delta \dot{q}$ and $\Delta \dot{R}$. The initial covariance matrix could be set as Eq.11. 


$$
\mathbf{P}(0)=\left[\begin{array}{cccccc}
\Delta q^{2} & 0 & 0 & 0 & 0 & 0 \\
0 & \Delta \dot{q}^{2} & 0 & 0 & 0 & 0 \\
0 & 0 & \Delta R^{2} & 0 & 0 & 0 \\
0 & 0 & 0 & \Delta \dot{R}^{2} & 0 & 0 \\
0 & 0 & 0 & 0 & 0 & 0 \\
0 & 0 & 0 & 0 & 0 & 0
\end{array}\right]
$$

And time-to-go could be derived from Eq.12.

$$
\hat{t}_{g o}=\hat{R} / \hat{\dot{R}}
$$

\section{Optimal guidance law}

Let assume that the autopilot had an order dynamics, and the transfer function from command acceleration $a_{m c}$ to response acceleration $a_{m}$ was as Eq.13. Let $a_{t}$ be acceleration of target.

$$
\frac{a_{m}(s)}{a_{m c}(s)}=\frac{1}{s / \omega_{m}+1}
$$

The intercept model was as Fig.3.

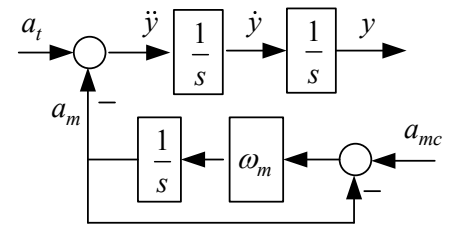

Figure 3. Model of optimal guidance law

The state equation of the optimal guidance law could be derived from Fig. 3 as Eq. 14 .

$$
\left[\begin{array}{c}
\dot{y} \\
\ddot{y} \\
\dot{a}_{t} \\
\dot{a}_{m}
\end{array}\right]=\left[\begin{array}{cccc}
0 & 1 & 0 & 0 \\
0 & 0 & 1 & -1 \\
0 & 0 & 0 & 0 \\
0 & 0 & 0 & -\omega_{m}
\end{array}\right]\left[\begin{array}{c}
y \\
\dot{y} \\
a_{t} \\
a_{m}
\end{array}\right]+\left[\begin{array}{c}
0 \\
0 \\
0 \\
\omega_{m}
\end{array}\right] a_{m c}
$$

With the miss distance was zero and minimum control energy, the optimal guidance law could be obtained after some mathematics. The optimal guidance law was as Eq.15.

$$
a_{m c}=N^{\prime}\left[\frac{y}{t_{g o}^{2}}+\frac{1}{t_{g o}} \dot{y}+0.5 a_{t}-\frac{1}{T^{2}}\left(e^{-T}-1+T\right) a_{m}\right]
$$

Where

$$
N^{\prime}=\frac{T^{2}\left(e^{-T}-1+T\right)}{-\frac{1}{2} e^{-2 T}-2 T e^{-T}+\frac{1}{3} T^{3}-T^{2}+T+\frac{1}{2}} \quad T=\omega_{m} t_{g o}
$$

When under small angle assumption, Eq.16 could be derived.

$$
q_{p} \approx \frac{y}{\dot{R} t_{g o}}
$$

Took the derivative of Eq.16, then Eq.17 could be easily obtained.

$$
\dot{q}_{p}=\frac{y+\dot{y} t_{g o}}{\dot{R} t_{g o}^{2}}
$$

Substituted Eq.17 into Eq.15, and with the estimate information, the optimal guidance law could be written in LOS coordinate system as Eq.18.

$$
a_{m c}=N^{\prime} \hat{\dot{R}} \hat{\dot{q}}_{p}+0.5 N^{\prime} \hat{a}_{t}^{l}-N^{\prime} \frac{1}{T^{2}}\left(e^{-T}-1+T\right) a_{m}^{l}
$$

\section{Three loop acceleration autopilot}

In the pitch plane, the linear dynamics equations of flight vehicle could be written as Eq.19.

$$
\left\{\begin{array}{l}
\dot{\alpha}=-b_{\alpha} \alpha+\omega_{z}-b_{\delta} \delta \\
\ddot{\vartheta}=-a_{\alpha} \alpha-a_{\omega} \dot{\vartheta}-a_{\delta} \delta \\
a_{y}=V(\dot{\vartheta}-\dot{\alpha})
\end{array}\right.
$$

Where $\alpha$ was angle of attack, $\delta_{e}$ was angle of elevator, $\dot{\vartheta}$ was body angular rate, $V$ was speed of vehicle, $a_{y}$ was lateral acceleration. $b_{\alpha}, b_{\delta}, a_{\alpha}, a_{\omega}$ and $a_{\delta}$ were dynamics coefficients whose definition were given in reference [6].

The transfer function of $\delta_{e}$ to $a_{y}$ was as Eq.20.

$$
\begin{aligned}
\frac{a_{y}(s)}{\delta_{e}(s)} & =-V \cdot \frac{-b_{\delta} s^{2}-a_{\omega} b_{\delta} s+\left(a_{\delta} b_{\alpha}-a_{\alpha} b_{\delta}\right)}{s^{2}+\left(a_{\omega}+b_{\alpha}\right) s+\left(a_{\omega} b_{\alpha}+a_{\alpha}\right)} \\
& =K_{a} \cdot \frac{A_{2} s^{2}+A_{1} s+1}{T_{m}^{2} s^{2}+2 \xi_{m} T_{m} s+1}
\end{aligned}
$$

The transfer function of $\delta_{e}$ to $\alpha$ was as Eq.21.

$$
\begin{aligned}
\frac{\alpha(s)}{\delta_{e}(s)} & =-\frac{b_{\delta} s+\left(a_{\omega} b_{\delta}+a_{\delta}\right)}{s^{2}+\left(a_{\omega}+b_{\alpha}\right) s+\left(a_{\alpha}+a_{\omega} b_{\alpha}\right)} \\
& =k_{\alpha} \frac{B_{1} s+1}{T_{m}^{2} s^{2}+2 \xi_{m} T_{m} s+1}
\end{aligned}
$$

The transfer function of $\delta_{e}$ to $\dot{\vartheta}$ was as Eq.22.

$$
\begin{aligned}
\frac{\dot{\vartheta}(s)}{\delta_{e}(s)} & =-\frac{a_{\delta} s+\left(a_{\delta} b_{\alpha}-a_{\alpha} b_{\delta}\right)}{s^{2}+\left(a_{\omega}+b_{\alpha}\right) s+\left(a_{\omega} b_{\alpha}+a_{\alpha}\right)} \\
& =K_{\dot{g}} \frac{\left(T_{i} s+1\right)}{T_{m}^{2} s^{2}+2 \xi_{m} T_{m} s+1}
\end{aligned}
$$

$K_{a} 、 T_{m} 、 \xi_{m} 、 T_{\alpha}$ were function of $a_{\omega} 、 a_{\alpha}$ 、 $a_{\delta_{e}}, b_{\alpha}, b_{\delta}$, whose definitions were also given in reference [6].

The transfer function of actuator was as Eq.23.

$$
\frac{\delta_{e}(s)}{\delta_{e c}(s)}=\frac{1}{T_{d}^{2} s^{2}+2 T_{d} \mu_{d} s+1}
$$

Three-loop acceleration autopilot with pseudo-angle of attack feedback had great robustness [1], which could be used to make sure the flight vehicle operate well. The diagram of the three-loop acceleration autopilot was as Fig.4. 


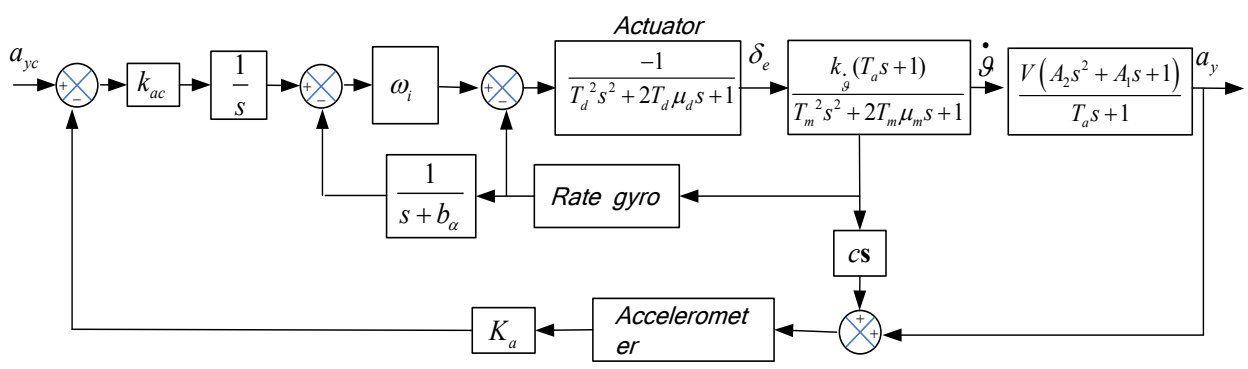

Figure 4. Three-loop autopilot with pseudo-angle of attack feedback

In Fig. $4, K_{a}, K_{a c}$ and $\omega_{i}$ were the design parameters of. Pole placement method was usually used to design the autopilot. The expected poles of three-loop autopilot were a first order slow root and a pair of second-order fast roots, such as Eq.24.

$$
\frac{a_{y}(s)}{a_{y c}(s)}=\frac{\left(A_{2} s^{2}+A_{1} s+1\right)}{(\tau s+1)\left(T_{m}{ }^{* 2} s^{2}+2 \xi_{m}{ }^{*} T_{m}{ }^{*} s+1\right)}
$$

With the inner loop feedback which usually was called as pseudo-angle of attack feedback, the characteristics of the flight vehicle was improved well. That seemed to be a new control model was obtained from the inner feedback. That was the course of robustness of three-loop acceleration autopilot.

For instance, if static stability coefficient $a_{\alpha}$ changed from -150 (static instability) to 150 (static stability), the bode diagram of open loop of three-loop autopilot could be obtained as Fig. 5 as $T_{m}{ }^{*}=0.05, \xi_{m}{ }^{*}=0.7, \tau=0.5$. The breakpoint of the open loop was at actuator input. At the crossover frequency, the magnitude and the phase values were very close for every $a_{\alpha}$.

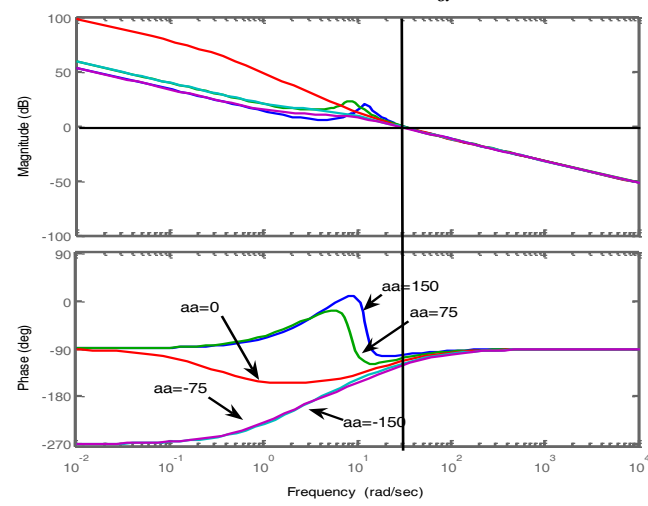

Figure 5.Bode diagram of open loop of three-loop autopilot

\section{Simulation}

To illustrate the performance of the proposed methods, the head-to-head intercept simulation was done, which was a very difficult condition. The optimal guidance law and the control method as well as extended Kalman filter were all used in the simulation as before.

The initial conditions of the simulation were listed in Table 1.The standard deviations of measurement noises were listed in Table 2.
Table 1. The initial conditions of the simulation

\begin{tabular}{|c|c|c|c|c|c|c|c|}
\hline $\begin{array}{c}q_{p 0} \\
\left({ }^{\circ}\right)\end{array}$ & $\begin{array}{c}\dot{q}_{p 0} \\
(\% / \mathrm{s})\end{array}$ & $\begin{array}{c}R_{0} \\
(\mathrm{~km})\end{array}$ & $\begin{array}{c}\dot{R}_{0} \\
(\mathrm{~m} / \mathrm{s})\end{array}$ & $\begin{array}{c}a_{m x 0}^{l} \\
\left(\mathrm{~m} / \mathrm{s}^{2}\right)\end{array}$ & $\begin{array}{c}a_{m y 0}^{l} \\
\left(\mathrm{~m} / \mathrm{s}^{2}\right)\end{array}$ & $\begin{array}{c}a_{t x 0}^{l} \\
\left(\mathrm{~m} / \mathrm{s}^{2}\right)\end{array}$ & $\begin{array}{c}a_{t y 0}^{l} \\
\left(\mathrm{~m} / \mathrm{s}^{2}\right)\end{array}$ \\
\hline 30 & 1.2 & 5 & -100 & 10 & 20 & 20 & 40 \\
\hline
\end{tabular}

Table 2. The standard deviations of measurement noises

\begin{tabular}{|c|c|}
\hline$\sigma_{q}\left({ }^{\circ}\right)$ & $\sigma_{R}(\mathrm{~m})$ \\
\hline 0.2 & 7 \\
\hline
\end{tabular}

The simulation results were showed as Fig. 6 to Fig.11. Fig.6 and Fig.7 was the estimate acceleration along and perpendicular to the LOS of the target. After a short transient time, the estimate result was very close to the real value. Fig.8 showed that although the angle measured by the seeker was noisy, the angle of LOS was estimated very well. The angular rate of LOS was estimated well as Fig.9 too. With the estimated guidance signal and the optimal guidance law, the command acceleration of the autopilot was obtained as Fig.10. The command acceleration was noisy especially at the transient time. After the transient time the command acceleration was smooth and concentrated to nearly zero at the end. Though the input was noisy, the three-loop autopilot with pseudo-angle of attack feedback worked well. The autopilot filtered the noise and tracked the command acceleration well as Fig.10. The angle of attack and the angle of elevator were as Fig.11, which satisfied the demand.

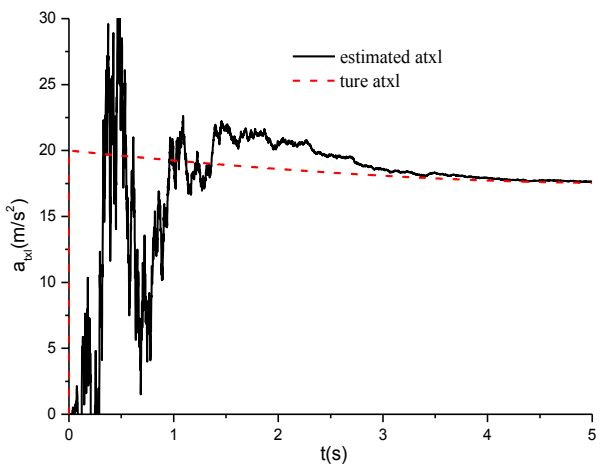

Figure 6.Estimated result of $a_{t x}^{l}$ 


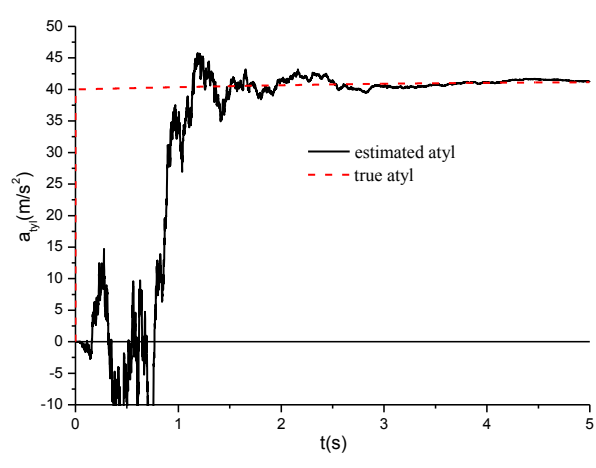

Figure 7. Estimated result of $a_{t y}^{l}$

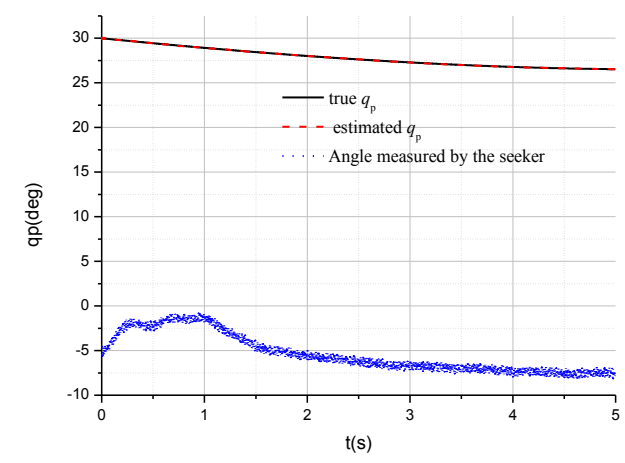

Figure 8.Estimated result of angle of LOS

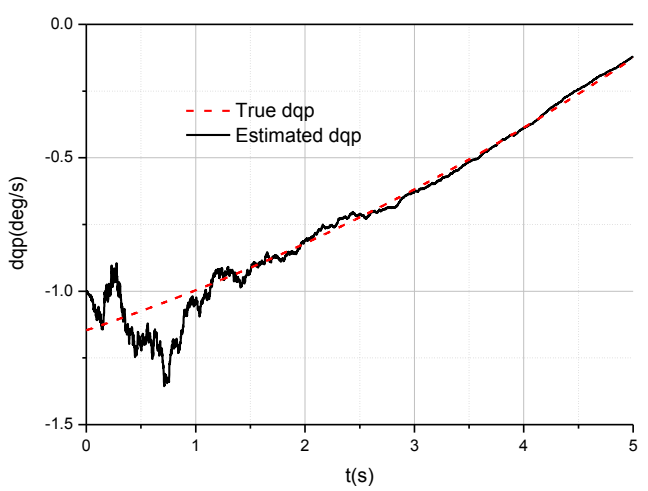

Figure 9.Estimated result of angular rate of LOS

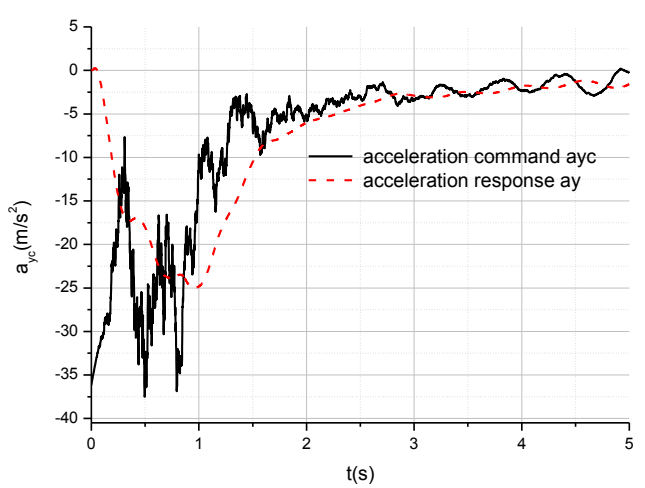

Figure 10. The input and output of the autopilot

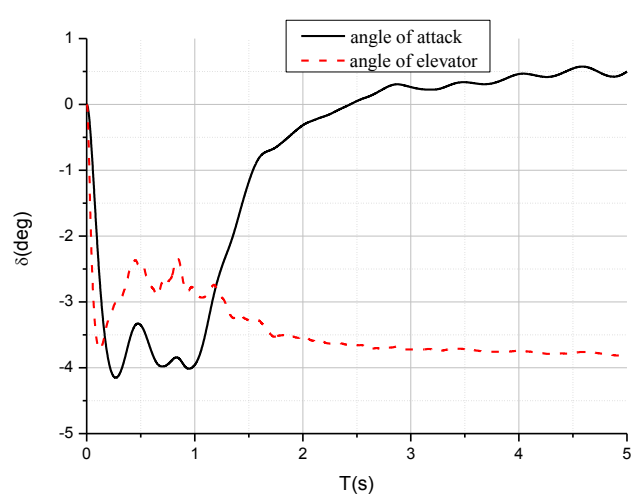

Figure 11.The angle of attack and angle of elevator

\section{Conclusion}

Based on the measurements of phased array radar seeker, the extended Kalman filter has been designed in the LOS coordinate system to estimate the maneuvering target acceleration, time-to-go, and angular rate of LOS. Also the practical optimal guidance law was derived in LOS coordinate system. The three-loop autopilot with pseudoangle of attack feedback was proposed, which had strong robustness. From the simulation, it could be showed that after a short transient time, the estimated guidance signal could converge to their real values. The command acceleration obtained from optimal guidance law was noisy at the transient time, after the transient time it was much smooth and finally concentrated to nearly zero at the end. Three-loop autopilot worked well, which filtered the noise and tracked the command acceleration well. The proposed guidance and control method could reach the request of the flight vehicle with strap-down seeker.

\section{References}

1. Z. Qi. Design of tactical missile guidance and control system.CAPH Press, Beijing( 2018).

2. F. Li, Q. Xia, Z. Qi,J. Syst. Eng. Electron. 35 (8): 1717-1722( 2013).

3. K. S. Erer and O. Merttopcuoglu. J. Guid. Control Dynam., 35(2):700-704( 2012).

4. P. Zarchan. Fundamentals of Kalman Filtering: A Practical Approach third edition. AIAA, Virginia(2009).

5. F. Li, Sh.Jia, K.Bu . J. of Ordnance Equip. Eng. 39(12):20-24(2018).

6. Z. Qi, Q. Xia. Guided weapon control systems. Beijing Institute of Technology Press, Beijing,(2004).

7. P. Zarchan, Tactical and strategic missile guidance 5th editon. AIAA, Virginia (2007).

8. C. K. Ryoo, H. S. Shin, and M. J. Tahk, IEEE Trans. Aero. Electron. Sys., 46 (1): 80-95(2010).

9. G. Hexner, T. Shima .,IEEE Trans. Aerosp. Electron. Syst.43 (1) :71-78(2007).

10. P. Gurfil. Control Eng. Pract., 11(7):819-832( 2003).

11. Y. Oshman, D. Arad. J. Guid. Control Dynam., 27(4):595-606( 2004). 